\title{
A New Methodology for Blind Image Deconvolution
}

\author{
L. Jimson \\ Asst. Professor, Dept of CSE, \\ REC, Coimbatore,
}

\author{
P. Senthil \\ PG Scholar, Dept of CSE \\ CIET, Coimbatore,
}

\author{
D. Chandrakanth \\ PG Scholar, Dept of CSE \\ CIET, Coimbatore,
}

\begin{abstract}
In image processing, the elimination of noise is a highly difficult in research area. It is one of the most important powerful technologies that will form science and engineering in the twenty first century. The broad knowledge, digital image processing is any shape of information processing used for which both input and output are images, such as photographs. Image restoration method is mainly used to eliminate the inescapable distortions and noise that leave into an image at some point in the image capture process. Direct image restoration difficulty is briefly revisited subsequently a recent method based on inverse filtering for the perfect image restoration in noiseless case is proposed. Noisy case is addressed by means of introducing a regularization term into the objective function in order to avoid the noise amplification. The filter recognition problem is considered in the Multichannel context. A new strong solution to estimate the degradation matrix filter is then derived and used in combination with a total variation approach to restore the original image.
\end{abstract}

\section{General Terms}

Variational techniques, Image processing.

\section{Keywords}

Automatic Color Enhancement (ACE), Image Restoration, Human Visual System (HVS).

\section{INTRODUCTION}

The Images are captured by an image detection device such as digital cameras, Charge-Coupled Device (CCD) and mobile phones are regularly different than the actual image perceived by the device user. The main difference between the perceived and detected images are due to many factors such as the characteristics and settings of the image detection device, ambient lighting conditions, and the difference between the human visual perception model and the image detection device perception model, to name a not many. In image processing, one of the main problems is seen when detecting images is due to the uneven enlightenment of the image. This case, when the image discovery device detects an unevenly illuminated image, the resulting detected image will often have portions that are too light/dark such that image quality is significantly reduced. Previous art solution for evening out image enlightenment is by manual modification of image parameters. Designed for instance, the techniques known as evading and burning require a user to physically specify or identify regions of the image to be lightened or darkened.

Evading and burning consists of cutting and positioning physical masks during the photographic improvement process. This technique can also be carried out digitally by means of a mouse or other computer interface to select specific pixels or regions in an image to be lightened or darkened. An addition to physically identifying regions for adjustment, the user must also control the amount of adjustment. The problem with this technique is that it requires a certain level of skill and a large amount of time and effort to achieve. One more solution is based on edge-ratio preserving algorithms. Retinex or edgeratio preserving algorithms use an iterative processing of an image in order to determine a corrected output image. In this iterative method consists of taking ratios of a number of neighboring pixel values, multiplying these ratios and finally, resetting these ratios if some maximum value is achieved. This method image edge lightness values are better reproduced and, given a large enough number of ratios, these lightness values should be normalized relative to the image white. The hypothesis is that sequence of calculations mimics the human visual system. In particular, an observer is constantly scanning an image or sight and is performing some type of normalization relative to the sight or image white.

The algorithms attempt to model the human visual system (HVS) and reconstruct a digitally captured image with perceptually correct local color correction from a known scene. Given a calibrated input, this approach generates images that are similar to those that might be produced using the manual technique, where a calibrated input is an image that accurately represents some physical quantity that can be directly measured, such as radiance or luminance. However, current implementations tend to be computationally intensive.

There is no simple framework to allow an interactive or user specified control over these algorithms that is important for either correcting uncalibrated inputs images or for correcting under, over and unevenly exposed images. Additional approaches include histogram equalization, image segmentation, or specialized hardware. The histogram equalization method use properties of the statistical distribution of the lightness values in the image to find a quality correction curve for that image [17].

\section{THEORETICAL BACKGROUND}

In the Photoshop we can't get the clear image after applying color balance and Auto levels. It will affect the whole image and give them some unwanted image. The existing system did not take into account the spatial, local and nonlinear features of the Human Visual System (HVS), is part of the nervous system that allows you to see. Human Visual System reconstructs a world of three-dimensional objects from a twodimensional projection of that world. Human Visual System can only determine detail information. It is mainly used in small area of your vision center to do so. Human Visual System is a non-linear, non-uniformly and non-separable system. Though, with half-toning HVS can be model as a uniformed and linear system to help with assessing image quality.

We take into account the automatic color enhancement (ACE) equation in discrete terms and we show that it can be obtained as the gradient descent of certain energy functional. One side, by studying generalizations of the functional associated to ACE; we may understand which variational methods are suitable for color enhancement based on some properties of the Human Visual System. On the other side, the particular form of the ACE energy functional reveals explicit features of the model which are intrinsic in the discrete framework. The 
Compressed images not only reduce a web page's uploading and downloading time. The image takes up less space on the server in terms of space and bandwidth Superior compression performance.

Image segmentation techniques try to automatically partition images into regions. These regions are then lightened or darkened based on a study of these regions. Hardware such as logarithmic Charge-Coupled Device (CCD) sensors and dual sampling Complementary metal-oxidesemiconductor (CMOS) sensors provide a better means of capturing high dynamic range scene data. However, this hardware does not specify how this high dynamic range data should be processed or mapped to lower dynamic range devices. Categorize to "correct" images when they are captured by the detection device the images are initially converted into a digital data format to allow for image enhancement and processing. The digital data format is represented by an array of picture elements (i.e., pixels) where each pixel has associated digital information describing the image at that pixel location within the array. The digital data of the image can be represented in different types of color space representations. One type of color space representation, referred to as a perceptual color space (or color space model) representation, defines pixels by perceptual parameters of the image.

The color space model is distinct as a viewing specific transformation of the XYZ representation to/from a perceptual attributes representation. For example, in case of the CIELAB each color component is defined by three attributes lightness $\left(\mathrm{L}^{*}\right)$, chroma and hue. The coordinates $\mathrm{a}^{*}$ and $\mathrm{b}^{*}$ are the Cartesian coordinates that can be computed from lightness, chroma and hue using standard polar to Cartesian coordinate transformations. The $\mathrm{a}^{*}$ and $\mathrm{b}^{*}$ axes correspond to the redgreen and yellow-blue opponent color axes.

An additional state of the art perceptual color space or color appearance model that has been recently developed referred to as CIECAM97s defines color components by three attributes including lightness $(\mathrm{J})$, chroma $(\mathrm{C})$ and hue $(\mathrm{H})$. The use of ordinary and partial differential equations (PDEs) and variational techniques for image processing became a major research topic in recent years. One of the reasons for such interest is the fact that functional methods allow us to deal with several problems of image processing at the same time, thanks to the natural possibility of combining different types of algorithms in the framework of PDEs or variational techniques [17].

Moreover, making explicit the energy functional underlying a given algorithm may reveal relevant properties related to important visual information carried by an image, which may be not very easy to point out only with the analysis of the basic (discrete) formulae. Finally, the functional procedures can be implemented with efficient numerical methods that give highly accurate results. The contrast modifications induced by the minimization of the energy functional considered in the aforementioned paper did not take into account the spatial, local and nonlinear features of the Human Visual System (HVS), which are instead the guiding lines of perception models.

These characteristics are implemented in a recent perceptual inspired color correction algorithm: automatic color enhancement (ACE) in Fig 1 is the combination of the gradient descent technique for the minimization of the ACE functional with a numerical approximation of the related Euler-Lagrange equation allows us to rewrite the model in such a way that its computational complexity decreases from the original to, being the number of pixels in the image being processed. In practice, this means that high quality perceptualbased color corrected versions of high resolution images may be obtained in a matter of seconds, instead of tens of minutes, with a regular $\mathrm{PC}(\mathrm{P} 4,3 \mathrm{GHz})$.

\section{PRE-PROCESSOR}

The aim of all color perception algorithms is to map, for every fixed chromatic channel, the colorimetric intensity of a pixel into the corresponding perceptual quantity, called lightness after Land and McCann and indicated with in this present paper. The recombination of the lightness in all channels gives the triplet that estimates the color appearance of the pixel. We note that, since the goal of the model that we present here is image enhancement, the lightness may correspond qualitatively but not necessarily quantitatively to the perceptual correlate.

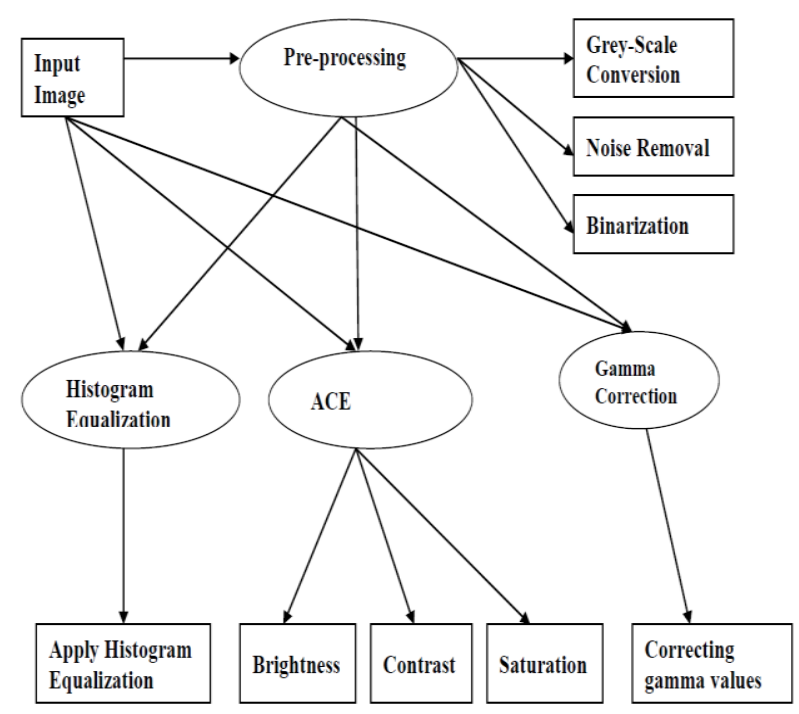

Fig 1: Pre-processor

It consist of three paramount important characteristics of the Human Visual System that every perceptual-inspired model should implement: the first is locality of color perception, i.e., the fact that the HVS is strongly influenced by the global and local context in which an observed scene lies [12]-[14]; the second is the spatial nature of the color perception, meaning that the actual sensation image induced by the HVS follows from a comparison of local information coming from different image areas [16]; finally, the HVS exhibits nonlinear responses both to global and local variations of the stimuli coming from an observed scene.

- This project is a perceptual based color correction of digital images in the framework of variational techniques.

- In this project we introduce novel image functional whose minimization produces a perceptually inspired color enhanced version of the original image.

- The perceptual technique permits a more flexible of contrast adjustment and brightness to digital images.

- We will use a numerical implementation of the gradient descent technique applied for color enhancement, a particular perceptual-based model of color enhancement. 


\section{METHODOLOGY}

The proposed approach has five modules namely ModifiedHuffman, Lossy compression, Manually Filtering Image Changes, Expand and resizing every picture, Compress saved images.

\subsection{Modified-Huffman}

In computer science and information theory, Modified Huffman coding is an entropy encoding algorithm used for image compression. The term refers to the use of the variable length code table for encoding a source symbol where the variable-length code table has been derived in a particular way based on the approximate probability of incidence for each possible value of the source symbol. Modified Huffman coding uses a specific method for choosing the representation for each symbol, resulting in a prefix-free code that express the most common characters using shorter strings of bits than or used for less common source symbol. Modified Huffman was able to design the most efficient compression method of this type: no other mapping of individual source symbols to same strings of bits will make a smaller average output size when the actual symbol frequencies agree with those used to create the code. A process was later found to do this in linear time if input probabilities (also known as weights) or sorted.

For asset of symbols with a uniform probability distribution and a number of members which is a power of two, Modified Huffman coding is equivalent to simple binary block encoding e.g. ASCII-coding .Modified Huffman coding in Fig 2 is such a wide spread method for creating prefix-free codes that the team "Modified Huffman Code" is widely used as a synonym for "prefix-free code" even when such a code is not produced by Modified Huffman algorithm. Though Modified Huffman coding is optimal for a symbol-By-Symbol code with an identified input probability distribution, its optimality can sometimes accidentally be over-stated. Both these methods can combine an arbitrary number of symbols for more efficient coding and generally adapt to the actual input statistics, the later of which is useful when input probabilities or not precisely known.

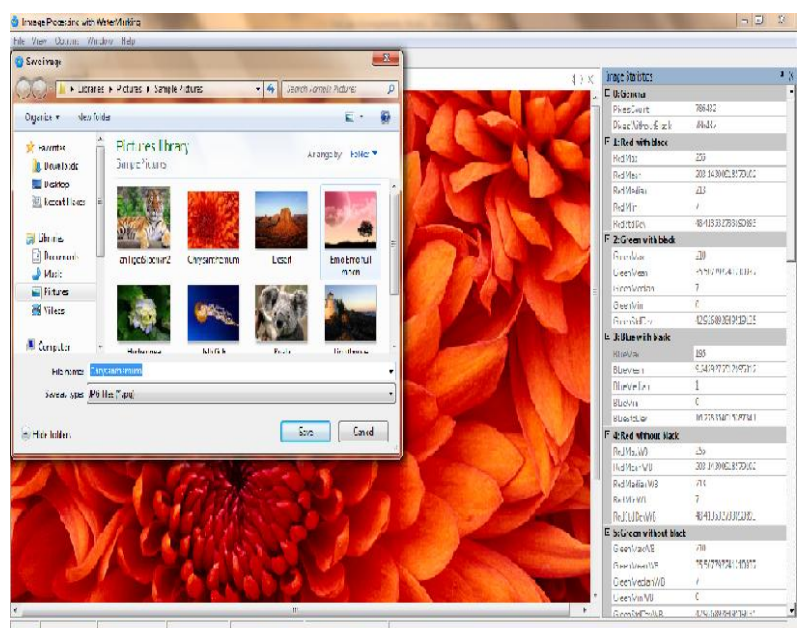

Fig 2: Modified-Huffman

\subsection{Lossy Compression}

Lossy compression is the case when binary data such as executables, documents are compressed. They require to be exactly reproduced when decompressed. However, images (and music too) need not be reproduced 'exactly'. A rough calculation of the original image is enough for the majority purposes as long as the error between the original and the compressed image is tolerable. The increasing use of digitized images, particularly on the Internet, has led to the need to compress such imagery to allow economical storage and fast data transfer.

A digital image, or "bitmap", consists of a grid of dots, or "pixels", with each pixel defined by a numeric value that gives its color. Images are defined as either "realistic", meaning photographs or realistic artwork with many shades and few straight lines, or "non-realistic", meaning graphs, block diagrams, cartoons and graphics banners with block colors and many straight lines. The RLE schemes are easy and fast, but their compression efficiency depends on the type of image data being encoded. Black-and-white image that is mostly white, such as the pages of a book, will encode extremely well due to the large amount of contiguous data that is all the same color in Fig 3. Image with a lot of colors that is very busy in appearance, however, such as a photograph, will not encode very well. Since the difficulty of the image is expressed as a large number of different colors because of this difficulty there will be relatively few runs of the same color.

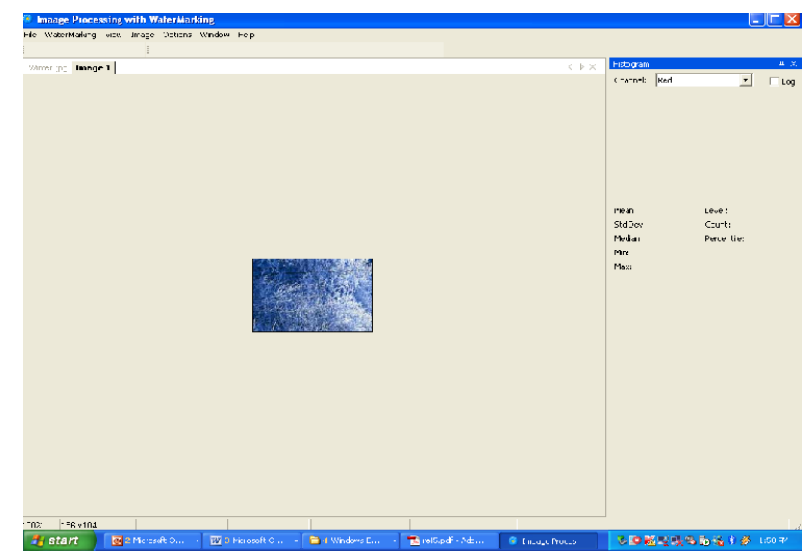

Fig 3: Lossy compression

\subsection{Manually Filtering Image Changes}

Manually filter the image such as shown in Fig 4.

- Colour changes, HSL \& YCbCr filter changes.

- Picture Binarization (Threshold, Sierra, Stucke).

- Picture Correlation and Convolution(Edges, Mean, Blur).

- Picture Morphology (Erosion, Opening, Closing).

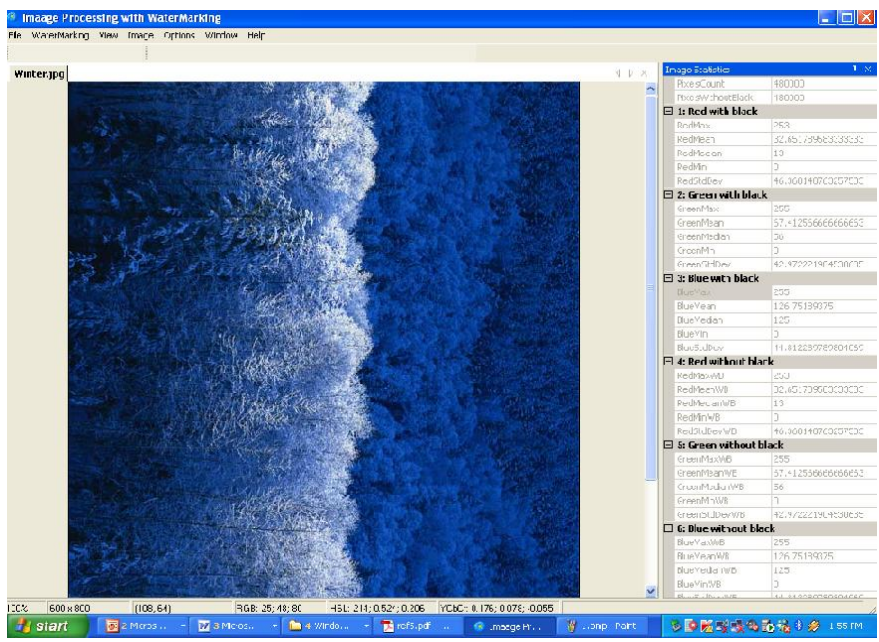

Fig 4: Manually Filtering Image Changes 


\subsection{Expand and resizing every picture}

- Manually change the image size(with zooming option)

- Pixel(picture element) of the picture height and width can be changed

- To change picture homogeneity and difference.

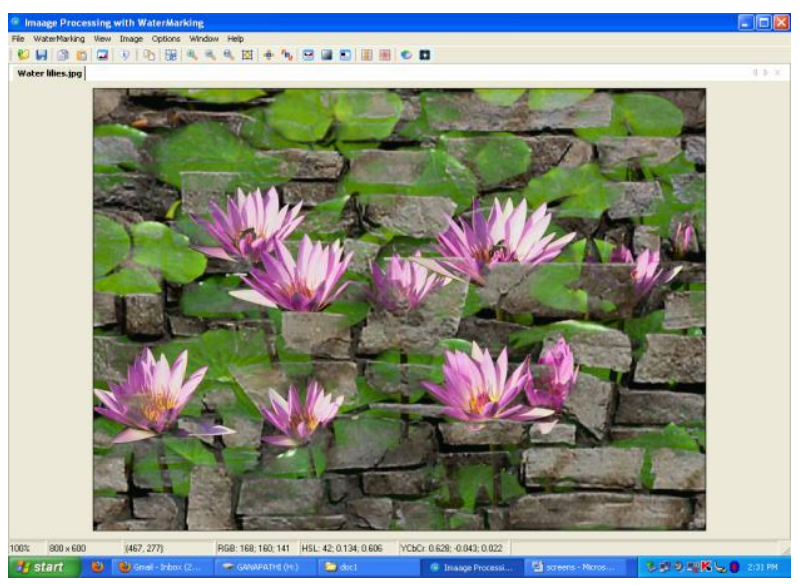

Fig 5: Expand and resizing every picture

\subsection{Compress saved images}

Image Compression is used to reduce size of picture. The compression software let's used for digitally compress large images, which saves time in opening and manipulating the images the program shown in Fig 6, can be used to compress an image of any size, while yielding better resolution of image compression.

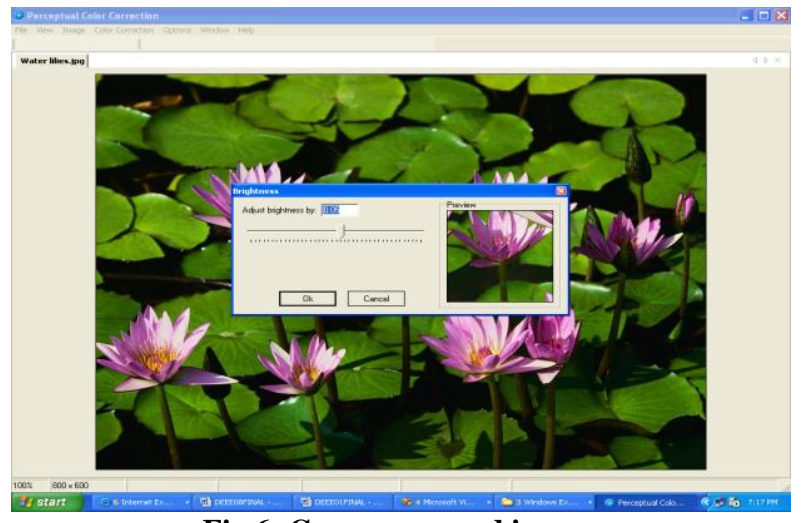

Fig 6: Compress saved images

\section{CONCLUSION AND FUTURE ENHANCEMENT}

This paper propose a new method for blind image deconvolution, examine some known approaches, and provide a new Multichannel method for restoring blurred and noisy images. Image restoration stands to benefit greatly by use of technologies. We have investigated global and local properties of color correction algorithms from the viewpoint of variational techniques. In particular, we focused our attention on the variational formulation of Automatic Color Enhancement (ACE), a color correction model that takes into account some of the most important features of human color perception. The variational formulation of ACE allowed us to clarify its global and local characteristics, which were somewhat hidden in its basic formulation. Furthermore, the approximation of the continuous variational formulation of ACE using a polynomial approximation leads to a significant reduction of the corresponding algorithm complexity, decreasing from without introducing significant changes in the results. This allows a very fast implementation.

Every application has its own merits and demerits. The project has covered almost all the requirements. Further requirements and improvements can easily be done since the coding is mainly structured or modular in nature. Changing the existing modules or adding new modules can append improvements. Future enhancements can be made to the application, so that the web site functions very attractive and useful manner than the present one.

\section{REFFERENCES}

[1] G. A. Showman and J. H. McClellan, "Blind polarimetric qualization of ultrawideband synthetic aperture radar imagery," presented at the Int. Conf. Image Processing, 2000.

[2] A. Jalobeanu, L. Blanc-Féraud, and J. Zerubia,"” "Hyperparameter estimation for satellite image restoration using a mcmc maximum likelihood method,"

Pattern Recognit., vol. 35, pp. 341-352, 2002.

[3] Y. V. Zhulina, "Multiframe blind deconvolution of heavily blurred astronomical images," Appl. Opt., vol. 45 , no. 28 , pp. $7342-7352$, Oct. 2006.

[4] S. Filip, ' S. Stanislava, F. Jan, and S. Tomá s, "Multichannel blind deconvolution of the shortexposure astronomical images," in Proc. Int.Conf. Pattern Recognition, Sep. 2000, vol. 3, pp. 53-56.

[5] M. Vrhel and B. L. Trus, "Multi-channel restoration of electron micrographs," in Proc. Int. Conf. Image Processing, Oct. 1995, vol. 2, pp. 516-519.

[6] H. J. Trussel, M. I. Sezan, and D. Tran, "Sensitivity of color LMMSE restoration of images to the spectral estimation," IEEE Trans. Signal Process, vol. 39, no. 1, pp. 248-252, Jan. 1991

[7] U. A. Al Suwailem and J. Keller, "Multichannel image identification and restoration using continuous spatial domain modeling," presented at the Int. Conf. Image Processing, Oct. 1997.

[8] F. Sroubek and J. Flusser, "Multichannel blind deconvolution of spatially misaligned images," IEEE Trans. Image Process., vol. 14, no. 7, pp. 874-883, Jul. 2005.

[9] N. P. Galatsanos, A. K. Katsaggelos, R. T. Chin, and A. D. Hillery, "Least squares restoration of multichannel images," IEEE Trans. Acoust., Speech, Signal Process., vol. 39, no. 10, pp. 2222-2236, Oct. 1991.

[10] F. Sroubek and J. Flusser, "Multichannel blind iterative image restoration," IEEE Trans. Image Process., vol. 12, no. 9, pp. 1094-1106, Sep. 2003.

[11] C. A. Ong and J. A. Chambers, "An enhanced NASRIF algorithm for blind image deconvolution," IEEE Trans. Image Process., vol. 8, no. 7, pp. 988-992, Jul. 1999.

[12] W. Souidene, K. Abed-Meraim, and A. Beghdadi, "Deterministic techniques for multichannel blind image deconvolution," presented at the Proc. ISSPA, Aug. 2005.

[13] L. Tong and Q. Zhao, "Joint order detection and blind channel estimation by least squares smoothing," IEEE 
Trans. Signal Process., vol. 47, no. 9, pp. 2345-2355, Sep. 1999.

[14] E. Fishler, A. Haimovich, R. Blum, D. Chizhik, L. Cimini, and R.Valenzuela, "MIMO radar: An idea whose time has come," presented at the IEEE Radar Conf., Apr. 2004.

[15] B. Hassibi, B. M. Hochwald, and T. L. Marzetta, "Multi-antenna wireless communications-From theory to algorithms," presented at the IEEE ICASSP, May 2002.

[16] D. G. Karakos and P. E. Trahanias, "Generalized multichannel imagefiltering structures," IEEE Trans. Image Process., vol. 6, no. 7, pp. 1038-1045, Jul. 1997.

[17] Wided Souidene, Karim Abed-Meraim, Azeddine Beghdadi "A new look to multichannel blind image deconvolution" IEEE TRANSACTIONS ON IMAGE PROCESSING, VOL. 18, NO. 7, JULY 2009.

\section{AUTHORS PROFILE}

L.Jimson is working as an Assistant Professor in Ranganathan Engineering College (R.E.C), Coimbatore. He received his M.E degree in Computer Science and Engineering at Coimbatore Institute of Engineering and Technology (C.I.E.T), Coimbatore and received his B.E degree in Computer Science and Engineering at Cape Institute of Technology, Nagarcoil. His Research areas are Image Processing and Networking.

P.Senthil is currently pursuing M.E, Computer Science and Engineering in Coimbatore Institute of Engineering and Technology (C.I.E.T), Coimbatore and received his B.Tech degree in Information Technology at Dhanalakshmi Srinivasan Engineering College, Perambalur. His Research areas are Networking, Image Processing and Cloud Computing.

Chandrakanth.D is currently pursuing M.E, Computer Science and Engineering in Coimbatore Institute of Engineering and Technology (C.I.E.T), Coimbatore and received his B.Tech degree in Information Technology at DMI College of engineering, Chennai. His Research areas are Natural language processing, Image Processing and Data Mining 